\title{
O USO DE IMUNOSSUPRESSORES: EM PACIENTES ADULTOS DE TRANSPLANTE RENAL
}

\author{
Sara Elen de Araujo Corado Silva ${ }^{1}$ \\ Alcione Silva de Carvalho ${ }^{2}$
}

RESUMO: Fármacos imunossupressores atuam reduzindo a resposta imunológica, porque existem situações em que o organismo não age da forma que deveria, e sendo assim reconhece elementos que não teria necessidade. $O$ transplante renal é uma opção terapêutica de escolha para o paciente com uma doença renal crônica, se for comparado com a diálise, o transplante tem melhor sobrevida, qualidade de vida, e até mesmo menor custo no longo prazo. Este trabalho visa apontar com clareza como o uso desses fármacos podem ter benefícios e malefícios, saber como é importante o uso correto da medicação, E como o profissional Farmacêutico deve fazer a atenção a este paciente, utilizando dados para resolver essas questões com base em literaturas como Scielo, Google Acadêmico.

Palavras chaves: Imunossupressores. Transplantes Renais. Pacientes Adultos. Doença renal.

ABSTRACT: Immunosuppressive drugsact by reducingthe immune response, because there are situations in which the body does notact as it should, and thus recognizes elements that it would not need. And kidney transplantationis a therapeutic option of choice for the patient with a chronic kidney disease, if compared to dial ysis, transplantation has better survival, quality of life, and evenlowercost in thelongrun. This work ims to clearly point out how the use of these drugs canhave benefits and harms, as the Pharmacists hould care for this patient, using data to resolve theseis sues basedon literaturesuch as Scielo, Google Academic.

Key words: Immunosuppressants. KidneyTransplants. AdultPatients. Kidneydisease.

\section{INTRODUÇÃO}

O transplante é a transferência de um tecido ou órgão de um local para outro na mesma pessoa, ou entre diferentes pessoas (Ioannidou\&Makris, 2003).A terapia da

\footnotetext{
I Graduação do curso de Farmácia da Universidade Iguaçu - UNIG. E-mail: araujosara93o@gmail.com.

${ }^{2}$ Licenciatura e Bacharelado em Química com orientação tecnológica industrial e Doutora em Ciências pela UFRJ - Universidade Federal do Rio de Janeiro. Professora e Orientadora da UNIGUniversidade Iguaçu. Email: alcionecarvalhoıs7o@gmail.com.
} 
imunossupressão adequada é de suma importância para evitar rejeição acentuada de transplantes de órgãos, e assim evitando a perda de enxerto, porém doses não controladas e/ou doses elevadas desses medicamentos levam a toxicidade. (AGARWAL et. Al 2008)

Os imunossupressores agem de forma contrária ao que se pensa, pois eles são utilizados para reduzir a resposta imune do nosso organismo, para que não se rejeite o órgão transplantado que no caso deste estudo é o rim. Como também por exemplo pode ocorrer reação de hipersensibilidade, as alergias, e doenças auto imunes como o Lúpus, exatamente por consequência da resposta imunológica querendo expulsar algum corpo estranho do organismo. (DENTON et.al 1999).

O Brasil é o segundo país em número completo de transplantes renais. No período de 54 anos foram realizados 107.836 transplantes renais, sucedendo a um crescimento anual permanente, inclusive pelo alto crescimento de transplantes com rim de doador falecido. ( MEDINA-PESTANA et.al 20II). Estima-se que atualmente, em torno de 64 mil pacientes estejam em acompanhamento com enxerto renal funcionante. O país é uma referência mundial de financiamento público dos transplantes, sendo a maioria realizada pelo Sistema Único de Saúde (SUS), desde a conjectura prétransplante, cirurgia, acompanhamento e divisão dos medicamentos imunossupressores. ( GARCIA 2019).

O diagnóstico da doença renal é estabelecido por critérios clínicos e laboratoriais. Uma vez firmado a diagnose, tornam-se obrigatórias a pesquisa da causa da nefropatia, independentemente da faixa etária do paciente, e a avaliação do grau de comprometimento funcional. (HARDINGER K, BRENNAN DC 2002). Os métodos mais utilizados para estimar a função renal abrangem a determinação do nível da creatinina sérica, da depuração da creatinina e da ureia e equações que estimam a função renal embasada na concentração sérica e urinária da creatinina e da ureia, na devida ordem. (VELLA J 20II).

O desígnio da purificação da creatinina é a mais utilizada. A equabilidade de Cockcroft-Gault permite a aferição da taxa de filtração glomerular (eTFG). A fórmula utilizada é a da depuração de creatinina $=[($ I40-idade $) \times$ peso $(\mathrm{kg})] /[$ creatinina sérica $(\mathrm{mg} / \mathrm{dL})$ x 72]. (LUBOWITZ $\mathrm{H}$ et.al 1976). Para mulheres, tende-se multiplicar o 
resultado por 0,85 com atributo da menor massa muscular. Além disso, é também aconselhado ajustar os dados obtidos pela superfície corporal. (RAMOS E, KLEIN CL 2012). A denotação de diálise ou transplante geralmente se faz quando a eTFG pertencer abaixo de $18 \mathrm{ml} /$ minuto. A sociedade canadense de transplante propôs que o transplante não deva ser feito a menos que a filtração glomerular seja menor que $20 \mathrm{ml} / \mathrm{m}$ e com sinais de declínio funcional nos últimos 6 a 12 meses. O protocolo da sociedade canadense indica que o transplante "preemptivo" (pré-dialítico) é o predileto e deve ser encorajado, não devendo também ser realizado com filtração glomerular maior que $20 \mathrm{~mL} / \mathrm{m}$.

Constituem os objetivos específicos estabelecer o estado de imunossupressão, referindo-se ao tratamento utilizado no momento do transplante, manter o estado de imunossupressão, prevenir o desenvolvimento e tratar os episódios de rejeição aguda e crônica. ( KNOLL G et.al 2005).

A expressão terapia de indução refere-se ao tratamento imunossupressor utilizado no período transoperatório e que pode se expandir por 07 a Io dias em âmbito hospitalar. Já a terapia de manutenção refere-se ao esquema imunossupressor utilizado posteriormente a este período.

Mas vale destacar que a identificação e o controle dos fatores de risco da insuficiência renal e do seu diagnóstico em estágio inicial, bem como o encaminhamento ágil e condizente para o atendimento especializado dão à Atenção Básica um caráter essencial para um melhor resultado terapêutico e prognóstico dos casos, inclusive quanto aos transplantados.

\section{METODOLOGIA}

Trata se de uma revisão da literatura que teve consultas realizadas em artigos científicos. Para a construção dessa pesquisa foi feito buscas de dados Scielo, Google Acadêmico. Os termos utilizados foram Imunossupressores, Transplante renal, e resposta imunológica. 


\section{JUSTIFICATIVA}

É de suma importância que o leitor entenda alguns fatores, como por exemplo, o porquê é utilizado os imunossupressores, pois muitos pacientes não acreditam que o fármaco irá ajudar no tratamento. Pois visam muito os efeitos colaterais. E este trabalho tem como designo manifestar que o uso correto é eficaz ao paciente

\section{DESENVOLVIMENTO}

\section{DIAGNÓSTICO}

$\mathrm{Na}$ significação do risco imunológico do transplante é indicado que seja avaliada antes da realização do procedimento para a definição do esquema de indução. A definição tem embasamento nas características demográficas de quem doa e de quem é o receptor e fatores de risco comprovadamente associados com maior risco de rejeição incluindo: (HU et.al 2009).

I. Baixa conformidade HLA (Human leukocyte antigen)

2. Ocorrências de abrandar os antígenos HLA através de gestações, transfusões de sangue e transplante prévio;

3. A assiduidade de anticorpos pré-formados específicos contra antígenos HLA do concessor;

4. Distúrbio inicial do enxerto. 
FIGURA I: fases que vão de I (menos grave) a 5 (mais grave) da doença.

\section{FASE 1

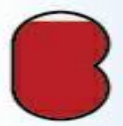 \\ Os rins ainda funcionam bem, \\ mas aparece já algum sinal de lesão, \\ como proteína na urina.}

FASE 2

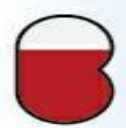

Os rins têm uma leve diminuição no seu funcionamento. Assim como na fase 1 , importante ter o diagnóstico de DRC, pois é possível prevenir as fases seguintes com o tratamento adequado

\section{FASE 3}

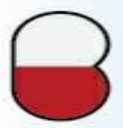

Os rins perdem sua eficiência de maneira moderada e já começam a dar os primeiros sinais de doença. Se ainda não começou, este é o momento de iniciar um tratamento com o nefrologista

FASE 4

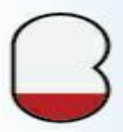

O funcionamento renal já está bastante prejudicado e exige mais atenção e cuidados

\section{FASE 5}

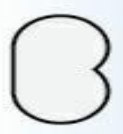

Os rins não funcionam mais. É quando deve-se iniciar a Terapia Renal Substitutiva (diálise ou transplante renal)

Fonte: Grupo Chr.net

\section{DIAGNOSE DE REJEIÇÃO AGUDA}

A rejeição aguda de enxerto renal é de forma generalizada, determinada como uma degeneração aguda da incumbência da inserção associada às alterações histopatológicas definidas de adesão com a Classificação Internacional de Banff É importante distinguir que, diferentemente do que se conceituava no passado, quando se consideravam apenas variações da creatinina sérica acima de $20 \%$ ou $30 \%$, em relação ao valor basal do paciente, nos dias de hoje a ascensão renitente acima de ıo\% já é eficiente para se considerar a hipótese de rejeição.

Nessas situações, recomenda-se a reiteração da dosagem da creatinina sérica a cada sete dias para afirmação ou não da conversão observada. Se confirmada, todos os esforços devem ser feitos para embargar a progressão do quadro.

Já que a perda funcional pode ser ocasionada secundária a outras justificações, como nefrotoxicidade por inibidores da calcineurina, anormalidades urológicas ou vasculares e 
utilização de diuréticos, sendo decidido pelo nefrologista esclarecer sobre a necessidade de biópsia renal. (Group KDIGOKTW 2009).

\section{MANUTENÇÃO DA IMUNOSSUPRESSÃO}

É de suma importância e idealidade que exista associação de um inibidor da calcineurina ou tacrolimo com uma droga anti proliferativa como azatioprina ou micofenolato de mofetila ou sódico, ou um corticosteroides como por exemplo a prednisona.

\section{ESQUEMAS DE MANUTENÇÃO DA IMUNOSSUPRESSÃO}

É necessário que seja individualizado o regime de imunossupressores dependendo das combinações do concessor receptor.

FIGURA 2: Esquema de atuação no interior da célula, dos diferentes fármacos

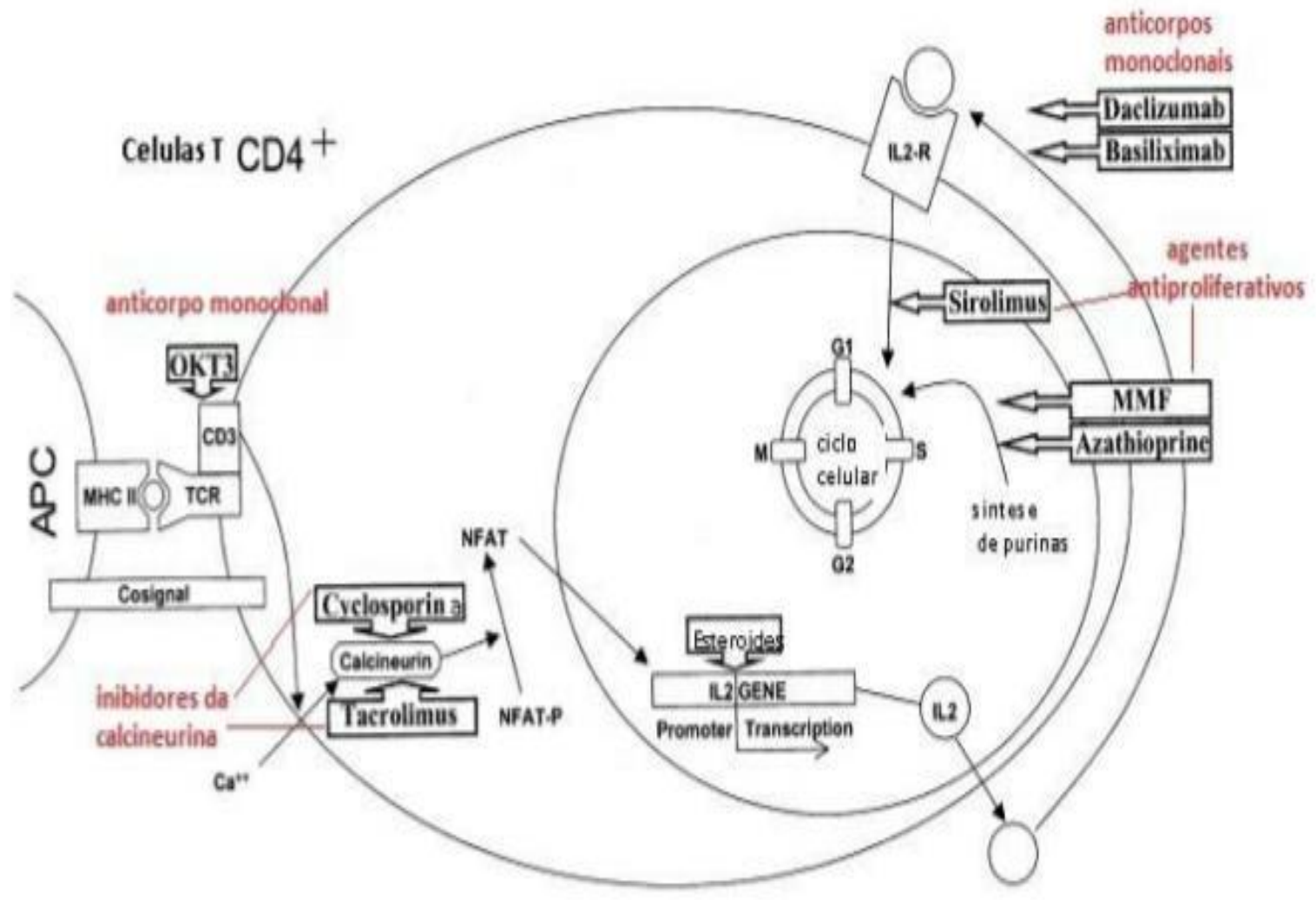

Fonte: adaptado de Mueller, 2004. 


\section{ALGUMAS COMBINAÇÕES POSSÍVEIS}

\section{I Tacrolimo + Micofenolatos}

$\mathrm{Na}$ prevenção de rejeição aguda celular tem eficácia elevada e interposto por antircorpos, e para pacientes de alto risco é usado para o tratamento como padrão. Quando é usada esta combinação a concentração sanguínea de tracolimo deve ser mantida entre 4 -IIng/mL

\subsection{Tacrolimo + Azatioprina}

Essa combinação é ideal para o paciente que tem baixo risco imunológico, este regime proporciona a qualidade do enxerto e do paciente a longo prazo.

\subsection{Tacrolimo + Inibidor mTOR}

Existem evidências que esta combinação é mais eficaz do que o tracolimo + micofenolatos na prevenção da rejeição aguda. Sendo importante se atentar a interação farmacocinética e farmacodinâmica entre essas classes de fármacos que resulta em maior risco de nefrotoxicidade e acometendo comprometimento da função renal.

\subsection{Regime livre de corticosteroídes}

Nas combinações citadas acima, são usadas corticosteroídes na sua composição. Este regime pode ser utilizado em pacientes com baixo risco imunológico sendo aqueles que provavelmente teria reações adversas, como crianças, idosos, obesos, diabéticos, a retirada desses fármacos pode ser realizado em 5 a 7 dias após o transplante ou uma remoção mais tardia que pode ocorrer entre 3 a 12 meses após o mesmo.

\section{TRATAMENTO DA REJEIÇÃO AGUDA}

Para este tratamento é necessário a apresentação clínica e diagnóstico histologico. O conjunto terapêutico envolve metilprednisolona, termoglobulina, imunoglobulina humana e plasmaferese.

Mesmo existindo tratamento ideal, havendo rejeição deve se avaliar aderência do receptor de tranpslante renal ao regime de imunossupressão, sendo incluso as doses diárias e as concentrações sanguíneas. 


\section{PROCEDIMENTO DA REJEIÇÃO AGUDA CELULAR}

O procedimento de episódio de rejeição aguda celular compreende o uso de pulsoterapia com metilprednisolona e/ou termoglobulina.

É feito uma biopsia renal antes deste tratamento sempre que for necessário. A metilprednisolona está indicada como primeira escolha para este tratamento.

\section{TRATAMENTO DA REJEIÇÃO AGUDA INTERPOSTA POR ANTICORPOS}

A melhor escolha para tratar rejeição aguda medida por anticorpos, é feita com sessões de plasmaferese, associando a administração de imunoglobulinas humanas

\begin{tabular}{|lll|}
\hline Fármaco & Dosagem & Via de adiministração \\
\hline Ciclosporina & Cáps $10 / 25 / 50 / 100 \mathrm{mg}$ & Sol Oral e injetável \\
\hline Tracolimo & 1 e $5 \mathrm{mg} / 5 \mathrm{mg} / \mathrm{mL}$ & Sol Oral / Sol. Injetável \\
\hline Azatioprina & $50 \mathrm{mg}$ & VO \\
\hline Prednisona & 5 e $20 \mathrm{mg}$ & VO \\
\hline Prednisolona & $1 \mathrm{mg} / \mathrm{mL}$ e $3 \mathrm{mg} / \mathrm{mL}$ & Sol Oral \\
\hline Metilprednisolona & $500 \mathrm{mg}$ & Injetável \\
\hline Micofenolato de mofetila & $500 \mathrm{mg}$ & VO \\
\hline Micofenolato de sódio & 180 e $360 \mathrm{mg}$ & VO \\
\hline Sirolimo & Dragéas 1 e $2 \mathrm{mg}$ & VO \\
\hline Everolimo & $0,5 / 0,75 /$ e $1 \mathrm{mg}$ & VO \\
\hline Imunoglobulina humana & $\begin{array}{l}\text { Frasco ampola } \\
0,1 / 1,0 / 2,5 / 3,0 / 5,0 / 6,0 \mathrm{mg}\end{array}$ & Injetável \\
\hline Basiliximabe & Frasco ampola $20 \mathrm{mg}$ & Injetável \\
\hline $\begin{array}{l}\text { Imunoglobulina antitimócitos } \\
\text { humanos termoglobulima }\end{array}$ & Frasco ampola $25 / 100 / 200 \mathrm{mg}$ & Injetável \\
\hline
\end{tabular}

Quadro I: fármacos, e dosagens de imunossupressores.

Fonte: Próprio autor.

\section{ESQUEMA DE ADMINISTRAÇÃO DE ALGUNS FÁRMACOS}

\section{I Indução}

- Basiliximabe: É administrado $20 \mathrm{mg}$ IV no intra-operatório e mais $20 \mathrm{mg}$ IV no quarto dia pós operatório. Para crianças abaixo de $35 \mathrm{~kg}$ é recomendado doses de Iomg. 
- Metilprednisolona: Indicado 250 a Iooomg no intra-operatório. Já em crianças a dose é de apenas $30 \mathrm{mg} / \mathrm{kg}$. Sendo utilizado doses adicionais no pós operatório dependendo do protocolo do centro.

- Imunoglobulina humana: É prescrito $2 \mathrm{~g} / \mathrm{kg}$, sendo administrado em dose única ou dividida entre 2 a 5 dias dependendo do protocolo. É recomendado fraciona e a dose total em múltiplos de $\mathrm{ig} / \mathrm{kg}$ ou menos para diminuir efeitos adversos relacionados a infusão.

\section{Io. MANUTENÇÃO DE ALGUNS FÁRMACOS}

- Prednisona ou Prednisolona: Depende muito do protocolo do centro. É ideal iniciar com $0,5 \mathrm{mg} / \mathrm{kg}$ a $\mathrm{Img} / \mathrm{kg} /$ dia com ablactação gradual.

- Tacrolimo: Preescrito 0,05 a 0,15mg/kg de 12 em 12 horas para adultos, e dose de até $0,3 \mathrm{mg} / \mathrm{kg}$ inicialmente de 12 em 12 horas para crianças.

- Azatioprina: Segundo a bula, é recomendado de I a $4 \mathrm{mg} / \mathrm{kg} / \mathrm{dia}$ para pacientes com uso de regime imunossupressor triplo. (OGER, 2007; TAYLOR et al., 2005).

- Ciclosporina: Inicialmente e 3 a $6 \mathrm{mg} / \mathrm{kg}$ a cada 12 horas, por VO adequando-se a seguir acordo com a concentração sanguínea.

\section{TRATAMENTO DA REPULSÃO AGUDA}

- Metilprednisolona: Pode ser utilizado de 3 a 5 dias, de 5 a romg/kg IV

- Imunoglobulina Antitimócitos humanos (coelhos) timoglobulina: É usado por 7 a I4 dias, de I,omg/Kg/dia.

- Imunoglobulina Humana: Sobre a posologia não foram encontradas para o tratamento da rejeição aguda mediada por anticorpos são apenas indicadas doses de I00-500mg/kg em dias alternados após sessões de plasmaferese.

\section{BENEFÍCIOS ESPERADOS}

- Melhor qualidade de vida;

- Aditamento da sobrevida do paciente;

- Reduz taxas de refeições aguda crônicas. 


\section{MONITORAMENTO}

Para o sucesso do transplante renal, é necessário um cuidadoso método que se expande para a vida toda. A equipe médica deve ser cautelosa ao avisar ao paciente, e seus familiares que a cirurgia do transplante é apenas o começo desse tratamento e que se não for feito o processo corretamente poderá ser comprometido os resultados.

O paciente tem que ser monitorado por exames laboratoriais, consultas clínicas regulamente para que o médico acompanhe e veja se corre risco e efeitos adversos, infecciosos, e até mesmo diagnóstico precoce de eventos imunológicos. Se o paciente não demonstra complicações laboratoriais e clínicas o intervalo de visitas médicas e até de exame, deve ser gradativamente aumentado.

É recomendado para pacientes estáveis:

- 2 consultas por semana no $\mathrm{I}^{\circ}$ mês;

- No segundo e terceiro mês serão consultas semanais;

- Já no quarto e sexto mês são consultas quinzenais;

- É mensais no sétimo e décimo segundo meses;

- E bimestrais ou trimestrais depois do primeiro ano.

Tende-se levar em consideração que o médico juntamente com a equipe multidisciplinar e da família são de suma importância para que o medicamento seja corretamente utilizado. A supervisão a intervalos de pouca duração é necessária para um melhor controle.

Medicamentos imunossupressores têm distintas propriedades de efeitos colaterais e podem afetar a qualidade de vida. Para o médico escolher esses fármacos é importante informar aos pacientes sobre os medicamentos escolhidos e os diferentes efeitos colaterais e resultados que podem de alguma maneira afetar o bem estar do paciente. $O$ tratamento com os fármacos imunossupressores tem que ser mantido enquanto o paciente estiver vivo e enxerto funcionante. 


\section{CORTICOSTEROÍDES}

Deve-se monitorar clinicamente e laboratoriais e com a pressão arterial, glicemia e eletrólitos devendo ser realizado. Com intervalo definido por critério médico.

\section{CICLOSPORINA}

Monitoriza-se por meio de PA, exames laboratoriais, clínicos, realizações de hemograma, creatinina, lipídeos entre outros, devendo ser realizada com intervalo definido a critério médico.( SCHIFF J, COLE E, CANTAROVICH M 2003).

\section{SIROLIMO E EVEROLIMO}

É realizada dosagens séricas de glicose, lipídeos, devendo ser feito com intervalo que for decidido pelo médico, Alguns efeitos acontecer como edema pulmonar, impotência, cefaleia, derrame pleural, gengivite entre outros. (COLE E et.al 2003).

\section{INTERAÇÕES MEDICAMENTOSAS}

\section{CICLOSPORINA OU TACROLIMO}

Existem alguns fármacos que agem de forma negativa com a junção dos dois imunossupressores. Cetoconazol, anlodipino, fluconazol são uns que por exemplo podem aumentar o nível sanguíneo.

\section{SIROLIMO}

Quando administrada com indutores por exemplo anticonvulsionantes isonazida, rifampicina, e até com antifúngicos, antibióticos macrolideos pode causar interações consideráveis devendo ser feito ajuste de doses. (PAN et al., 2015).

Tratamento de suportes/profilaxias:

É necessário que o paciente siga alguns profilaxias após o transplante:

Ivermectina: sendo usado $200 \mathrm{mch} / \mathrm{kg} / \mathrm{dia}$ por 2 dias, ou albendazol $400 \mathrm{mg} / \mathrm{dia}$ por 3-5 dias para profilaxia de estrongiloidiase disseminada. 
Sulfametoxazol-trimeoprima 40o-8omg ao dia durante 6 meses para profilaxias de infecções por pneumocystis jirovecci para que se previna infecções urinárias e infecções por toxoplasma gondii.

\section{CONCLUSÃO}

Para que o tratamento independente de qual seja, para ser eficiente depende em larga escala da cooperação ativa do paciente, sendo que a adesão à terapia imunossupressora não pode ser dispensada para evitar complicações que influenciem de forma negativa a função do órgão e sobrevida do paciente logo após transplante. A aprovação ao regime terapêutico quase sempre não é uma tarefa simples de efetivação na rotina dos doentes; já existem estudos que apontam que 30 a 50\% dos doentes, não conseguem aderir ao regime terapêutico na sua totalidade. A adesão pode variar muito de acordo com o tipo de transplante, idade, sexo.

Atualmente combinação da terapia imunossupressora assegura baixos índices de rejeição aguda do transplante, diminuição das hipóteses de rejeição crónica e aumento da vida do transplante. Como se descreveu, uma variedade de diferentes agentes imunossupressores, tanto farmacológicos como biológicos estão agora disponíveis para uso clínico em transplante de órgãos sólidos e não só. (BRAHM, M. M. T. 2012).

A prioridade tem que ser de selecionar regimes imunossupressores que os efeitos secundários sejam diminuídos, especialmente para aqueles que têm um impacto negativo na homeostase do organismo. Novos avanços na terapia imunossupressora estão dependentes da investigação na área da indução de tolerância após a transplantação clínica, para se obterem melhores resultados sem afetar a qualidade de vida do paciente. É necessário que se observe os critérios para incluir e excluir os pacientes neste protocolo, bem como monitorar tratamento, verificando doses prescritas e dispensadas, e se está sendo adequado o uso daquela medicação. O paciente que faz um transplante de rim deve ser acompanhado periodicamente por profissionais capacitados, de preferência em serviços habilitados pelo sistema nacional de transplante. ( TAYLOR et.al 2005).

E o profissional farmacêutico tem como papel principal mostrar qual a dosagem foi prescrita pelo médico no qual o paciente deverá utilizar, por quanto tempo, e até 
mesmo quais reações adversas podem ocorrer, fazendo sua atenção farmacêutica, e assim pedindo para ser feito até mesmo um acompanhamento com o mesmo, para se saber, se o tratamento está sendo eficaz e correto para o paciente, caso o contrário é mais fácil de falar com o médico e assim podendo ser acordado um medicamento e posologia que melhor se encaixe ao mesmo.

\section{REFERÊNCIAS BIBLIOGRÁFICAS}

Agarwal, A., Shen, L. Y., \& Kirk, A. D. (2008). The role of alemtuzumab in facilitating maintenance immunosuppression minimization following solid organ transplantation. Transplant Immunology, 20

Denton MD, Magee CC, Sayegh MH. Immunosuppressive strategies in transplantation. Lancet. 1999;353(9158):1083-91.

Medina-Pestana JO, Galante NZ, Tedesco-Silva H, Jr., Harada KM, Garcia VD, AbbudFilho M, et al. Kidney transplantation in Brazil and its geographic disparity. J Bras Nefrol. 2011;33(4):472-84

Garcia VD KE, Abbud Filho M. Avaliação Econômica do Transplante Renal no Brasil. In: Silva Junior GB OJ, Martins CTB, editor. A Nefrologia e o Sistema de Saúde do Brasil. ı. São Paulo: Livraria Balieiro; 2019. p. 175-200.

Hardinger K, Brennan DC. Maintenance immunosuppressive therapy in renal transplantation in adults: UpToDate; 2002 [24 aug, 202I]. Available from: http://www.uptodate.com/contents/maintenance-immunosuppressivetherapy-in-renaltransplantation- in-adults.

Vella J, Brennan DC. Induction immunosuppressive therapy in renal transplantation in adults: UpToDate; $201 \mathrm{I}$ [26 aug, 202I ]. Available from: http://www.uptodate.com/contents/induction-immunosuppressive-therapy-in-renaltransplantation- in-adults.

Lubowitz H, Slatopolsky E, Shankel S, Rieselbach RE, Bricker NS. Glomerular filtration rate. Determination in patients with chronic renal disease. JAMA. 1967;199(4):252-6.

Cockcroft DW, Gault MH. Prediction of creatinine clearance from serum creatinine. Nephron. 1976;16(I):3I-4I. 
Ramos E, Klein CL. Evaluation of the potential renal transplant recipient: UpToDate;2012 [26 Aug, 2021].Available

from:http://www.uptodate.com/contents/evaluation-of-the-potential-renal-transplantrecipient.

Knoll G, Cockfield S, Blydt-Hansen T, Baran D, Kiberd B, Landsberg D, et al. Canadian Society of Transplantation consensus guidelines on eligibility for kidney transplantation. CMAJ. 2005;173(I0):118I-4.

Dussauze, H., Bourgault, I., Doleris, L.-M., Prinseau, J., Baglin, a, \& Hanslik, T.(2007). [Systemic corticosteroid treatment and risk of infectious diseases].La Revue de Médecine Interne / Fondée ... Par La Société Nationale Francaise de Médecine Interne, 28(12), 84I-5I.

Germani, G., Lazzaro, S., Gnoato, F., Senzolo, M., Borella, V., Rupolo, G., \& Burra, P. (20II). Nonadherent Behaviors After Solid Organ Transplantation. Transplantation Proceedings, 43(I), 318-323.

Hu, Y., Turner, M. J., Shields, J., Gale, M. S., Hutto, E., Roberts, B. L., \& Kaplan, J. M. (2009). Investigation of the mechanism of action of alemtuzumab in a human $\mathrm{CD}_{52}$ transgenic mouse model. Immunology, $128(2), 260-70$.

Group KDIGOKTW. KDIGO clinical practice guideline for the care of kidney transplant recipients. Am J Transplant. 2009;9 Suppl 3:SI-I55.

Schiff J, Cole E, Cantarovich M. Therapeutic monitoring of calcineurin inhibitors for the nephrologist. Clin J Am Soc Nephrol. 2007;2(2):374-84.

Cole E, Maham N, Cardella C, Cattran D, Fenton S, Hamel J, et al. Clinical benefits of neoral $\mathrm{C}_{2}$ monitoring in the long-term management of renal transplant recipients. Transplantation. 2003;75(12):2086-90.

Einecke G, Mai I, Fritsche L, Slowinski T, Waiser J, Neumayer HH, et al. The value of $\mathrm{C}_{2}$ monitoring in stable renal allograft recipients on maintenance immunosuppression. Nephrol Dial Transplant. 2004;19(I):215-22.

Brahm, M. M. T. (2012). Adesão aos imunossupressores em pacientes transplantados renais. Programa D E Pós-Graduação Ciências, Medicina, 15-40. 
Taylor, A. L., Watson, C. J. E., \& Bradley, J. A. (2005). Immunosuppressive agents in solid organ transplantation: Mechanisms of action and therapeutic efficacy, Oncology/ Hematology, 56, 23-46.

Pan, Q., Xu, Q., Boylan, N. J., Lamb, N. W., G Emmert, D., Yang, J.-C., \& Hanes, J. (2015). Corticosteroid-loaded biodegradable nanoparticles for prevention of corneal allograft rejection in rats. Journal of Controlled Release: Official Journal of the Controlled Release Society, 201, 32-40.

Oger, J. (2007). Immunosuppression: promises and failures. Journal of the Neurological Sciences, 259(I-2), 74-8. 